\title{
Effect of Pd Nanoparticle Size on the Catalytic Hydrogenation of Allyl Alcohol
}

\author{
Orla M. Wilson, ${ }^{\ddagger}$ Marc R. Knecht, ${ }^{\dagger}$ Joaquin C. Garcia-Martinez, ${ }^{\ddagger}$ and Richard M. Crooks ${ }^{*}, \dagger$ \\ Department of Chemistry and Biochemistry, The University of Texas at Austin, 1 University Station, \\ A5300, Austin Texas 78712-0165, and Department of Chemistry, Texas A\&M University, P.O. Box 30012, \\ College Station, Texas 77842-3012
}

Received December 3, 2005; E-mail: crooks@cm.utexas.edu

We report that the rate of hydrogenation of allyl alcohol is a function of the diameter of the Pd nanoparticles (1.3-1.9 nm) used to catalyze the reaction. Furthermore, kinetic data indicate that this effect is electronic in nature for particles having diameters $<1.5$ $\mathrm{nm}$, but for larger particles it depends primarily on their geometric properties. This is a significant finding, because it represents a particle size effect for hydrogenation over unsupported Pd nanoparticle catalysts in a size range that has not been widely studied.

Dendrimer templates have been used to exert a high degree of control over the size, composition, and structure of catalytically active nanoparticles in the $<3 \mathrm{~nm}$ size range. ${ }^{1}$ Because of this, dendrimer-encapsulated nanoparticles (DENs) are well-suited for studying the effect of particle size on catalytic function. ${ }^{1}$ We have previously briefly noted a qualitative link between the size of DENs and their catalytic properties.,3 This paper represents our first quantitative analysis of the origin of these trends.

The procedure used to prepare Pd DEN catalysts has been previously reported. ${ }^{2}$ For the experiments reported here, the total moles of Pd used for each hydrogenation reaction was maintained constant for all experiments, but the $\mathrm{Pd} /$ dendrimer ratio was varied to yield DENs having different sizes. Specifically, sixth-generation, hydroxyl-terminated polyamidoamine dendrimers $(\mathrm{G} 6-\mathrm{OH})$ were used to synthesize Pd DENs containing an average of 55, 100, 147, 200 , or $250 \mathrm{Pd}$ atoms $\left(\mathrm{G} 6-\mathrm{OH}\left(\mathrm{Pd}_{n}\right)\right.$, where $n$ is the average number of atoms per particle). These particles have measured (by TEM, Figures S1 and S2, Supporting Information) and calculated (in parentheses) diameters of 1.3 (1.2), 1.4 (1.4), 1.5 (1.6), 1.7 (1.8), and 1.9 (1.9) nm, respectively. ${ }^{4,5}$

Alkene hydrogenation occurs via the Horiuti-Polanyi mechanism, which involves dissociative adsorption of $\mathrm{H}_{2}$ onto the catalyst surface, followed by stepwise hydrogenation of the $\mathrm{C}=\mathrm{C}$ double bond. ${ }^{6}$ Here, the rate of hydrogenation of allyl alcohol was determined by measuring hydrogen uptake. ${ }^{7}$ Briefly, $20.0 \mathrm{~mL}$ of the catalyst solution $(3.0 \mu \mathrm{mol}$ of Pd) was transferred to a Schlenk flask. The system was sealed and purged with $\mathrm{H}_{2}$ for 10 min and then stirred for an additional $10 \mathrm{~min}$. Allyl alcohol was added, and differential $\mathrm{H}_{2}$ pressure measurements were obtained every $10 \mathrm{~s}$ for $10 \mathrm{~min}^{7}$ Turnover frequencies (TOFs, $\mathrm{mol} \mathrm{H}_{2} / \mathrm{mol}$ active siteh) were determined from the slope of plots of turnover (mol $\mathrm{H}_{2} /$ mol catalyst) versus time.

Figure 1a shows that the rate of hydrogen consumption increases significantly as the measured particle diameter increases. However, this relationship does not correlate to a simple change in the total number of surface Pd atoms. This is because the total amount of Pd used in all these experiments is fixed, and therefore the number of catalyst particles present is smaller for the larger particles. This point is illustrated in Figure 1b. Here, the total number of Pd atoms present at particular locations on cuboctahedra are plotted as a

\footnotetext{
$\dagger$ The University of Texas at Austin.

* Texas A\&M University.
}
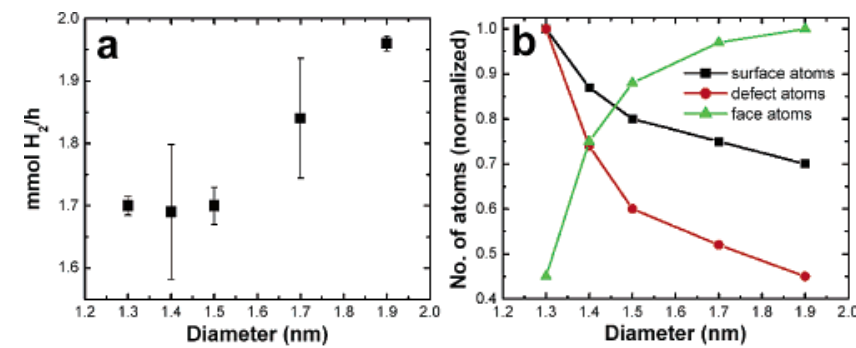

Figure 1. (a) Plot of the rate of hydrogen consumption as a function of particle diameter. (b) Plot of the total, calculated (Table S2, Supporting Information) numbers of surface, defect, and face atoms for each particle size. The data are normalized to the largest number of each type of atom.

function of the size of the cuboctahedron. Figure $1 \mathrm{~b}$ shows that only the total number of face atoms increases with particle size, while the numbers of surface and defect atoms both decrease. This is because the decrease in the number of particles dominates the increasing number of surface and defect atoms per particle as the particle size increases.

As mentioned in the previous paragraph, the total amount of Pd is constant for each reaction, and therefore the number of catalyst particles decreases as the particle size increases. This could result in the TOF being at least partially controlled by transport rather than kinetics for solutions containing larger particles. To rule out this possibility, catalyst solutions of $\mathrm{G} 6-\mathrm{OH}\left(\mathrm{Pd}_{147}\right)$ having concentrations that span the range corresponding to the data in Figure 1a were prepared, and then hydrogenation rates were determined. The results (Figure S3a, Supporting Information) indicated that the reaction rates $\left(\mathrm{mol} \mathrm{H}_{2}\right.$ consumed/h) are independent of catalyst concentration. Therefore, the reaction is zero order with respect to the catalyst concentration, and the particle-size-dependent change in hydrogenation rate (Figure 1a) is not attributable to transport. At much lower catalyst concentrations $(<0.4 \mu \mathrm{M})$, however, transport does dominate kinetics and a lower TOF is observed (Figure S3b, Supporting Information). Importantly, a plot of ln$\left[a_{0} / a_{t}\right]$ (where $a_{0}$ and $a_{t}$ are the substrate concentrations at time $=$ 0 and $t$, respectively) vs time (Figure S3c) results in a straight line, indicating that the reaction is first order with respect to the concentration of allyl alcohol. ${ }^{8}$

Differences in reaction rates as a function of catalyst size arise from either electronic or geometric effects. ${ }^{9}$ For example, as the size of a nanoparticle decreases, its electronic properties change from those of a metal to an insulator and then to something akin to a molecule. ${ }^{10,11}$ These changes modulate the catalytic properties of nanoparticles. Geometric effects are most evident when a reaction requires a specific type of surface atom, because the ratio of defect (vertex and edge) to face atoms changes dramatically as a function of size for $<5$-nm-diameter particles. While geometric effects have been observed for homogeneous colloidal Pd catalysts for both the Heck $^{12}$ and Suzuki ${ }^{13}$ coupling reactions, literature reports pertaining 


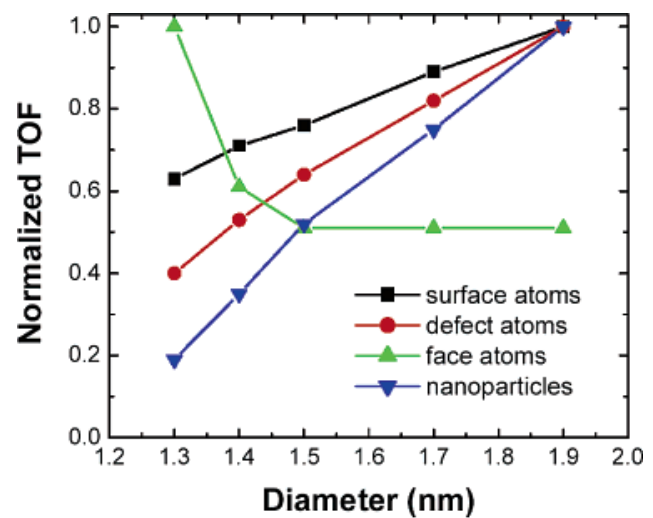

Figure 2. Plots of normalized TOF ( $\mathrm{mol} \mathrm{H}_{2} /(\mathrm{mol}$ active site-h)) as a function of particle diameter for the three different types of possible active sites and for the total number of nanoparticles.

to size effects in hydrogenation reactions focus almost exclusively on supported (heterogeneous) Pd catalysts. ${ }^{14-16}$ In addition to catalyst size, the preparation and structure of nanoparticles are also important factors that must be taken into account when comparing catalytic activity. This is because preparation methods, stabilizing ligands, and polydispersity can lead to activity changes that may mask true particle size effects. For example, Pd nanoparticles having similar diameters, but stabilized by either poly(vinylpyrrolidone) or 1,10-phenanthroline, exhibit very different catalytic activities for the hydrogenation of 1,3-cyclooctadiene. ${ }^{17}$

To determine the origin of the size effect shown in Figure 1a, we calculated the number of moles of surface, defect, and face atoms (Tables S2 and S3, Supporting Information), as well as the number of moles of Pd DENs, ${ }^{18}$ and then plotted the TOF, relative to each of these four parameters, vs nanoparticle size (Figure 2). That is, the TOF in Figure 2 is given in units of moles of $\mathrm{H}_{2}$ per mole of surface, defect, or face atoms (or number of particles) per hour, and then each data point is normalized to the highest TOF for each series. The absolute, measured values for the TOFs are provided in Table S1 and Figure S4 (Supporting Information). For three of the four plots, there is a monotonic increase in the TOF as the particle size increases. This indicates that, if the size effect shown in Figure 1a is due to geometric factors, the active site does not correlate to the number of surface atoms, defect atoms, or nanoparticles. However, when only face atoms are considered, the plot attains zero slope for particle diameters $\geq 1.5 \mathrm{~nm}$. Because this TOF is calculated in terms of the number of face atoms (mol $\mathrm{H}_{2} /$ mol face atoms-h), it means there is a $1: 1$ correspondence between the rate of hydrogen uptake and the number of face atoms for particles in this size range. This result is related to that shown in Figure 1b, which shows that, as the particle size increases, only the total number of face atoms in solution increases. From this result we conclude that, for Pd DENs having diameters in the range of $1.5-1.9 \mathrm{~nm}$, the hydrogenation of allyl alcohol occurs preferentially on the face atoms. Previous work focusing on ethene hydrogenation on model catalysts has indicated that the reaction is structureinsensitive and depends only on the number of Pd surface atoms available. ${ }^{19}$ Doyle et al. reported similar results for hydrogenation of ethene on $\mathrm{Pd} / \mathrm{Al}_{2} \mathrm{O}_{3}$ catalysts in $\mathrm{UHV}$, but catalyst size did affect the hydrogenation rate of trans-2-pentene under identical conditions. ${ }^{14}$ The latter observation was attributed to preferential hydrogenation of trans-2-pentene on face atoms. In our experiments, it is likely that changes in TOFs for smaller catalysts (diameters $<1.5 \mathrm{~nm}$ ) are dominated by differences in their electronic properties rather than the relative numbers of particular types of surface atoms. ${ }^{9}$

To summarize, we have shown a correlation between catalyst size and TOF for the hydrogenation of allyl alcohol. This structure/ function relationship is a direct consequence of the nanoscale size of the catalysts. The hydrogenation reaction is sensitive to both the electronic and geometric properties of the catalytic Pd nanoparticles, both of which change quickly in the 1.3-1.9-nm diameter size range. Our analysis indicates that the hydrogenation kinetics are dominated by electronic effects for the smallest particles $(<1.5$ $\mathrm{nm}$ diameter) and by geometric effects for larger particles (1.5$1.9 \mathrm{~nm}$ diameter). Results of the type described here are enabled by the high degree of monodispersity resulting from the dendrimer templating approach to nanoparticle synthesis. We recently discovered that this same templating approach can be used to control the composition ${ }^{3,20,21}$ and structure ${ }^{21}$ of metal nanoparticles, and therefore we are presently studying the effect of these variables on catalyst performance.

Acknowledgment. We gratefully acknowledge the U.S. Department of Energy, DOE-BES Catalysis Science grant no. DE-FG0203ER15471, the ACS Division of Analytical Chemistry (summer fellowship support for O.M.W.), the National Science Foundation (grant no. 0531030), and the Robert A. Welch Foundation for financial support of this work.

Supporting Information Available: Data tables, reaction rate, and TOF plots. This material is available free of charge via the Internet at http://pubs.acs.org.

\section{References}

(1) Scott, R. W. J.; Wilson, O. M.; Crooks, R. M. J. Phys. Chem. B 2005 , 109, 692-704

(2) Niu, Y.; Yeung, L. K.; Crooks, R. M. J. Am. Chem. Soc. 2001, 123, 68406846.

(3) Scott, R. W. J.; Wilson, O. M.; Oh, S.-K.; Kenik, E. A.; Crooks, R. M. J. Am. Chem. Soc. 2004, 126, 15583-15591.

(4) Scott, R. W. J.; Ye, H.; Henriquez, R. R.; Crooks, R. M. Chem. Mater 2003, 15, 3873-3878

(5) Daniel, M.-C.; Astruc, D. Chem. Rev. 2004, 104, 293-346.

(6) Horiuti, I.; Polanyi, M. Trans. Faraday Soc. 1934, 30, 1164-1172.

(7) Garcia-Martinez, J. C.; Wilson, O. M.; Knecht, M. R.; Crooks, R. M., manuscript in preparation.

(8) Laidler, K. J. Chemical Kinetics, 3rd ed.; HarperCollins Publishers: New York, 1987.

(9) Bond, G. C. Surf. Sci. 1985, 156, 966-981.

(10) Chen, S.; Murray, R. W.; Feldberg, S. W. J. Phys. Chem. B 1998, 102 9898-9907.

(11) Guo, R.; Murray, R. W. J. Am. Chem. Soc. 2005, 127, 12140-12143.

(12) Le Bars, J.; Specht, U.; Bradley, J. S.; Blackmond, D. G. Langmuir 1999, $15,7621-7625$.

(13) Li, Y.; Boone, E.; El-Sayed, M. A. Langmuir 2002, 18, 4921-4925.

(14) Doyle, A. M.; Shaikhutdinov, S. K.; Freund, H.-J. Angew. Chem., Int. Ed. 2005, 44, 629-631.

(15) Ryndin, Y. A.; Nosova, L. V.; Boronin, A. I.; Chuvilin, A. L. App. Catal. 1988, 42, 131-141

(16) Deganello, G.; Duca, D.; Martorana, A.; Fagherazzi, G.; Benedetti, A. J. Catal. 1994, 150, 127-134.

(17) Toshima, N.; Shiraishi, Y.; Teranishi, T.; Miyake, M.; Tominaga, T.; Watanabe, H.; Brijoux, W.; Bönnemann, H.; Schmid, G. Appl. Organomet. Chem. 2001, 15, 178-196.

(18) Benfield, R. E. J. Chem. Soc., Faraday Trans. 1992, 88, 1107-1110.

(19) Cremer, P. S.; Somorjai, G. A. J. Chem. Soc., Faraday Trans. 1995, 91 , $3671-3677$

(20) Scott, R. W. J.; Datye, A. K.; Crooks, R. M. J. Am. Chem. Soc. 2003 , $125,3708-3709$.

(21) Wilson, O. M.; Scott, R. W. J.; Garcia-Martinez, J. C.; Crooks, R. M. J. Am. Chem. Soc. 2005, 127, 1015-1024.

JA058217M 